\title{
Spectrum of Constitutive and Inducible Clindamycin Resistance in Staphylococcus Spp Isolated from Clinical Samples and Its Relation with Methicillin Resistance
}

\author{
Monika Rajani1* and Malay Banerjee ${ }^{2}$ \\ 'Department of Microbiology, Career Institute Of Medical Sciences \& Hospital, Lucknow \\ ${ }^{2}$ Professor \& Head, Career Institute Of Medical Sciences \& Hospital, Lucknow
}

\begin{abstract}
Backgound: Increasing Staphylococcal infections with changing patterns of antimicrobial resistance has led to renewed interest in macrolidelincosamide-streptogramin group B (MLSB) antibiotic. Misuse of MLSB antibiotics has increased resistance of Staphylococcus spp. to these drugs. Failure to detect inducible clindamycin resistance leads to therapeutic failure. Determine the prevalence of constitutive and inducible clindamycin resistance in clinical isolates of Staphylococcus spp. Ascertain the relationship between methicillin-resistance in Staphylococcus spp with constitutive and inducible clindamycin resistance.
\end{abstract}

Study Aimed: Study aimed to determine the prevalence of constitutive and inducible clindamycin resistance in clinical isolates of Staphylococcus spp. and ascertain the relationship between methicillin-resistance in Staphylococcus spp with constitutive andinducible clindamycin resistance.

Methods: Prospective study was carried over a period of six months with 722 clinical specimens. A total of 184 Staphylococcal isolates were identified. Staphylococcal isolates were speciated as S aureus, S epidermidis and S saprophyticus. Antibiotic susceptibility testing was done by Kirby-Bauer's disc diffusion method using cefoxitin, erythromycin, and clindamycin. A disc approximation test was performed for detection of inducible clindamycin resistance for all strains resistant to erythromycin but sensitive to clindamycin.

Results: Out of 184 staphylococcal isolates, 128 (69.5\%) S aureus and 56 (30.4\%) Coagulase negative Staphylococcus spp were identified. 114 $(61.9 \%)$ of the isolates were susceptible to both clindamycin and erythromycin. Overall $70(38.04 \%)$ isolates were resistant to erythromycin. Out of these,38 $(54.2 \% \%)$ of strains depicted cMLSB phenotype being resistant to both erythromycin and clindamycin.20 (28.5\%) isolates showed inducible clindamycin resistance while $12(17.1 \%)$ isolates indicated MS phenotype. Percentage of both constitutive and inducible clindamycin resistance was found to be higher in methicillin resistant staphylococcal isolates than methicillin sensitive isolates.

Conclusions: We recommend that the clinical microbiology laboratories should test the isolates for inducible clindamycin resistance by D test, for all isolates that appear erythromycin resistant and clindamycin susceptible in vitro.

Keywords: Clindamycin Resistance, Constitutive Resistance, Macrolide-Lincosamide-Streptogramin, Methicillin-Resistance, D-Test

\section{Introduction}

Staphylococcus aureus and coagulase negative staphylococcus spp are virulent pathogens that are currently significant etiology for a variety of infectious diseases. ${ }^{[1]}$ The antimicrobial resistance problem in methicillin resistant Staphylococcus aureus(MRSA) and methicillin resistant coagulase negative staphylococcus spp (MRCONS) isolates leads to high morbidity and mortality. ${ }^{[2]}$ Increasing frequency of such infections and changing patterns in antimicrobial resistance have led to renewed interest in the use of macrolide-lincosamide-streptogramin group $\mathrm{B}\left(\mathrm{MLS}_{\mathrm{B}}\right)$ antibiotics to treat such infections. ${ }^{[3]}$

Clindamycin is the preferred antibiotic for the treatment of methicillin resistant strains due to its excellent pharmacokinetic properties with optimum tissue penetration and high concentration in abscess. ${ }^{[4]} \mathrm{MLS}_{\mathrm{B}}$ antibiotics inhibit bacterial protein synthesis by binding to the $23 \mathrm{~S}$ rRNA of the $50 \mathrm{~S}$ ribosomal subunit.${ }^{[5]}$ However, the misuse of $\mathrm{MLS}_{\mathrm{B}}$ antibiotics has led to increased resistance of Staphylococcusspp. to these drugs due to a variety of resistance mechanisms. Ribosomal target site modification is the most common mechanism of resistance to $\mathrm{MLS}_{\mathrm{B}}$ in staphylococci. $\mathrm{MLS}_{\mathrm{B}}$ resistance can be either constitutive $\left(\mathrm{cMLS}_{\mathrm{B}}\right)$ or inducible $\left(\mathrm{iMLS}_{\mathrm{B}}\right){ }^{[6]}$

In vitro, isolates with constitutive expression are resistant to erythromycin and clindamycin due to presence of erm (erythromycin ribosome methylase) gene. In constitutive resistance, rRNA methylases are always produced unlike in inducible resistance where they are produced only in presence of an inducer. ${ }^{[7]}$ Isolates with inducible resistance are resistant to erythromycin but appear falsely susceptible to clindamycin in vitro. ${ }^{\left[{ }^{8]}\right.}$ Inducible clindamycin resistance is not recognized by using standard susceptibility test methods. ${ }^{[9]}$ Failure to identify iMLS $_{\mathrm{B}}$ may lead to clinical failure of clindamycin therapy. ${ }^{[9]}$ Apart from this, macrolide resistance can also be mediated by efflux mechanisms expressed by msr gene (MS phenotype), where only 
macrolides are resistant in vitro while clindamycin remains susceptible. ${ }^{[10]}$

The Clinical Laboratory Standards Institute (CLSI) has recommended the erythromycin -clindamycin disc approximation test (D-zone test) to detect the inducible clindamycin resistance. ${ }^{[1]}$ The aim of this study was to determine the prevalence of constitutive and inducible clindamycin resistance in clinical isolates of Staphylococcus spp in our region. There is scarcity of data regarding this prevalence from our geographical region. This will help avoid and clinical failure and retain the usefulness of clindamycin. We also tried to ascertain the relationship between methicillin-resistance in Staphylococcus spp with constitutive and inducible clindamycin resistance.

\section{Methodology}

This prospective observational study was conducted in the department of microbiology over a period of six months from January 2016 to June 2016 on the clinical samples from inpatient and outpatient departments. A total of 184 Staphylococcal isolates from 722 clinical specimens like pus, urine, blood, sterile body fluids, sputum, peripheral and central line catheter tips, wound swabs, respiratory aspirates were included in the study. Isolates were identified as Staphylococcus spp and further speciated as $\mathrm{S}$ aureus, $\mathrm{S}$ epidermidis and $\mathrm{S}$ saprophyticus by standard biochemical techniques like Gram staining, catalase test, slide and tube coagulase test, growth on Mannitol Salt agar and novobiocin $(5 \mu \mathrm{g})$ disc susceptibility testing. ${ }^{[12]}$ Antibiotic susceptibility testing was done on Mueller Hinton Agar (Himedia Laboratories Pvt. Ltd, India) by Kirby-Bauer's disc diffusion method using cefoxitin $(30 \mu \mathrm{g})$, erythromycin $(15 \mu \mathrm{g})$, and clindamycin $(2 \mu \mathrm{g})$. The zones were interpreted as per CLSI 2015 guidelines. ${ }^{[1]}$ Mec A mediated Oxacillin resistance was determined among the isolates using $30 \mathrm{ug}$ cefoxitin disc inoculated on Mueller Hinton Agar (Himedia Laboratories Pvt. Ltd, India) and incubating the plates at $33-35^{\circ} \mathrm{C}$ in ambient air for $16-18 \mathrm{hrs}$. The zones were interpreted as follows: sensitive $(\mathrm{S}) \geq 22 \mathrm{~mm}$, and resistant (R) $\leq 21 \mathrm{~mm}$ to cefoxitin, for Staphylococcus aureus; $\mathrm{S} \geq$ $25 \mathrm{~mm}$ and $\mathrm{R} \leq 24 \mathrm{~mm}$ to cefoxitin, for coagulase-negative Staphylococcus spp. ${ }^{[1]}$ The antibiotic discs were procured from Hi-media Laboratories Pvt. Ltd, India. Quality control (QC) was achieved with S. aureusATCC 25923 (American Type Culture Collection, Manassas, VA, USA).All isolates those were resistant to both erythromycin (zone size $\leq 13$ $\mathrm{mm}$ ) as well as clindamycin (zone size $\leq 14 \mathrm{~mm}$ ) were labeled under $\mathrm{CMLS}_{\mathrm{B}}$ phenotype. ${ }^{[8]}$

A disc approximation test was performed for detection of inducible clindamycin resistance for all strains that were resistant to erythromycin but sensitive to clindamycin as per CLSI 2015 guidelines. ${ }^{[1]} \mathrm{A} 2 \mu \mathrm{g}$ clindamycin disc was placed $15 \mathrm{~mm}$ away from the edge of a $15 \mu \mathrm{g}$ erythromycin disc. The culture plates were incubated aerobically overnight at $37^{\circ} \mathrm{C}$ and were examined under transmitted light the following day. The different phenotypes were appreciated as follows:

1. MS (Macrolide Streptogramin) phenotype: Such isolates were termed as D test negative.

2. Inducible MLSB (iMLS ${ }_{\mathrm{B}}$ ) phenotype: Such isolates were termed as D test positive. [Figure 1]

Statistical analysis was done with relevant tests. This study was approved by institutional ethics committee.

\section{Results}

Out of total 722 different clinical samples received, 184 staphylococcal isolates were identified. The distribution of positive samples according to the source of sample was found to be significantly unequal $(\mathrm{p}<0.001)$. The maximum positive samples were seen in swabs $(62.1 \%)$ followed by venous tips $(53.9 \%)$. [Table 1]

Out of the total positive isolates, $128(69.5 \%) \mathrm{S}$ aureus and 56(30.4\%) coagulase negative Staphylococcus spp were identified. Among $128 \mathrm{~S}$ aureus isolates, 84 (65.6\%) were phenotypically identified as MSSA while 44(34.3\%) as MRSA. Among 56 Coagulase negative staphylococcus spp, $48(85.7 \%)$ were phenotypically identified as $\mathrm{S}$ epidermidis while $8(14.2 \%)$ as S saprophyticus.

Among $48 \mathrm{~S}$ epidermidis strains, 38 (79.1\%) were phenotypically identified as MSSE, while $10(20.8 \%)$ as MRSE. Among $8 \mathrm{~S}$ saprophyticus isolates, all 4 isolates were methicillin sensitive.The distribution of MSSA according to the source of sample was found to be significantly unequal $(p<0.001)$ and same case were for MRSA, MSSE, MRSE and MSSS. [Table 1]

$114(61.9 \%)$ of the isolates were susceptible to both clindamycin and erythromycin. Overall 70(38.04\%) isolates were resistant to erythromycin.No significant relationship was found between the type of isolate and Erythromycin resistance $(\mathrm{p}=0.516)$. Out of the total erythromycin resistant strains,38 (54.2\%) of strains depicted $\mathrm{cMLS}_{\mathrm{B}}$ phenotype.20 (28.5\%) isolates showed inducible clindamycin resistance while $12(17.1 \%)$ isolates indicated MS phenotype.Statistically, the proportion of phenotype among the erythromycin resistant isolates was highly significant $(p<0.001)$.Percentage of both constitutive and inducible clindamycin resistance was found to be higher in methicillin resistant staphylococcal isolates than methicillin sensitive isolates. However statistically no significant relationship was found between the individual isolates and type of erythromycin resistance $(\mathrm{p}=0.136)$. [Figure 2, 3, Table 2] 
Table 1: Sample source and distribution of isolates of Staphylococcus spp.

\begin{tabular}{|c|c|c|c|c|c|c|c|c|}
\hline Source of sample & $\begin{array}{c}\text { Total No. of } \\
\text { samples }\end{array}$ & $\begin{array}{c}\text { Total samples } \\
\text { positive }\end{array}$ & MSSA & MRSA & MSSE & MRSE & MSSS & MRSS \\
\hline Urine & 257 & 16 & 8 & 0 & 0 & 0 & 8 & 0 \\
\hline Blood & 164 & 44 & 20 & 16 & 8 & 0 & 0 & 0 \\
\hline Pus & 137 & 60 & 28 & 16 & 12 & 4 & 0 & 0 \\
\hline Swabs & 58 & 36 & 16 & 10 & 6 & 4 & 0 & 0 \\
\hline Sputum & 39 & 4 & 2 & 2 & 0 & 0 & 0 & 0 \\
\hline Body fluids & 41 & 10 & 6 & 0 & 2 & 2 & 0 & 0 \\
\hline Venous tips & 26 & 14 & 4 & 0 & 10 & 0 & 0 & 0 \\
\hline Total & 722 & 184 & 84 & 44 & 38 & 10 & 8 & 0 \\
\hline Significance & $\begin{array}{l}\text { chi sq = 131 } \\
p<0.001\end{array}$ & $\begin{array}{l}\text { chi } s q=45.2 \\
p<0.001\end{array}$ & $\begin{array}{l}\text { chi sq } \\
=45.0 \\
p<0.001\end{array}$ & $\begin{array}{l}\text { chi sq } \\
=80.3 \\
p<0.001\end{array}$ & $\begin{array}{l}\text { chi sq } \\
=25.7 \\
p<0.001\end{array}$ & $\begin{array}{l}\text { chi sq } \\
=14.6 \\
p=0.023\end{array}$ & NA & \\
\hline
\end{tabular}

$M R S A=$ methicillin resistant S.aureus, $M S S A=$ methicillin sensitive S.aureus, MRSE = methicillin resistant $S$ epidermidis, MSSE = methicillin sensitive $S$ epedermidis, MSSS= methicillin sensitive $S$ saprophyticus, $M R S S=$ methicillin resistant $S$ saprophyticus

Table 2: MLSB phenotypes of Staphylococcal isolates and their relationship with methicillin resistance

\begin{tabular}{|c|c|c|c|c|c|}
\hline Isolates & Overall $\%$ of isolates & Erythromycin resistance (\%) & cMLS $_{\text {B }}$ Type & iMLS $_{B}$ Type & MS Type \\
\hline MSSA (84) & 45.7 & $32(38 \%)$ & $14(43.7 \%)$ & $10(31.2 \%)$ & $8(25 \%)$ \\
\hline MRSA (44) & 23.9 & $16(36.3 \%)$ & $10(62.5 \%)$ & $6(37.5 \%)$ & $0(0)$ \\
\hline MSSE (38) & 20.7 & $12(31.5 \%)$ & $6(50 \%)$ & $2(16.6 \%)$ & $4(33.3 \%)$ \\
\hline MRSE (10) & 5.4 & $6(60 \%)$ & $4(66.6 \%)$ & $2(33.3 \%)$ & $0(0)$ \\
\hline MSSS (8) & 4.3 & $4(50 \%)$ & $4(100 \%)$ & $0(0)$ & $0(0)$ \\
\hline MRSS (0) & 0.0 & 0 & 0 & 0 & 0 \\
\hline Total $(n=184)$ & - & $70(38.04 \%)$ & $38(54.2 \%)$ & $20(28.5 \%)$ & $12(17.1 \%)$ \\
\hline Significance & $\begin{array}{c}\text { chi } s q=3.26 \\
p=0.516\end{array}$ & & $\begin{array}{c}\text { chi sq }=12.4 \\
p=0.136\end{array}$ & & \\
\hline
\end{tabular}

Test of phenotype proportion : chi $s q=15.20, p<0.001$

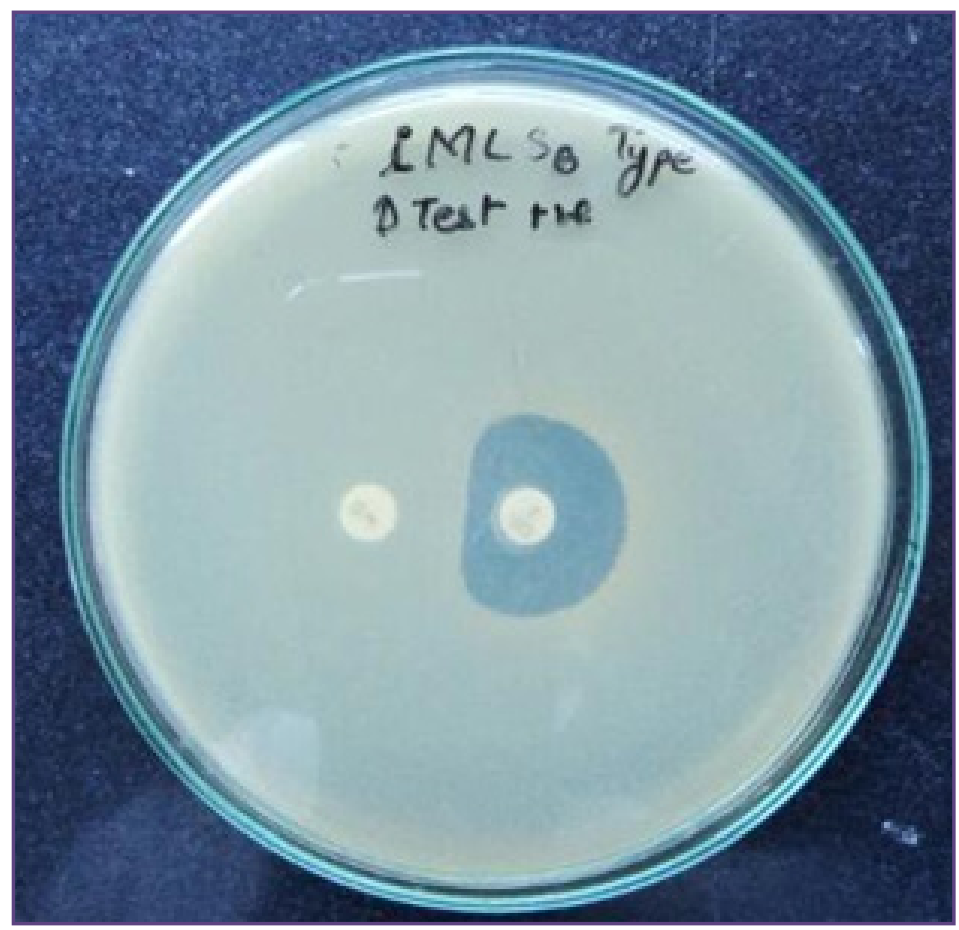

Fig. 1: The D- test showing positive result. 


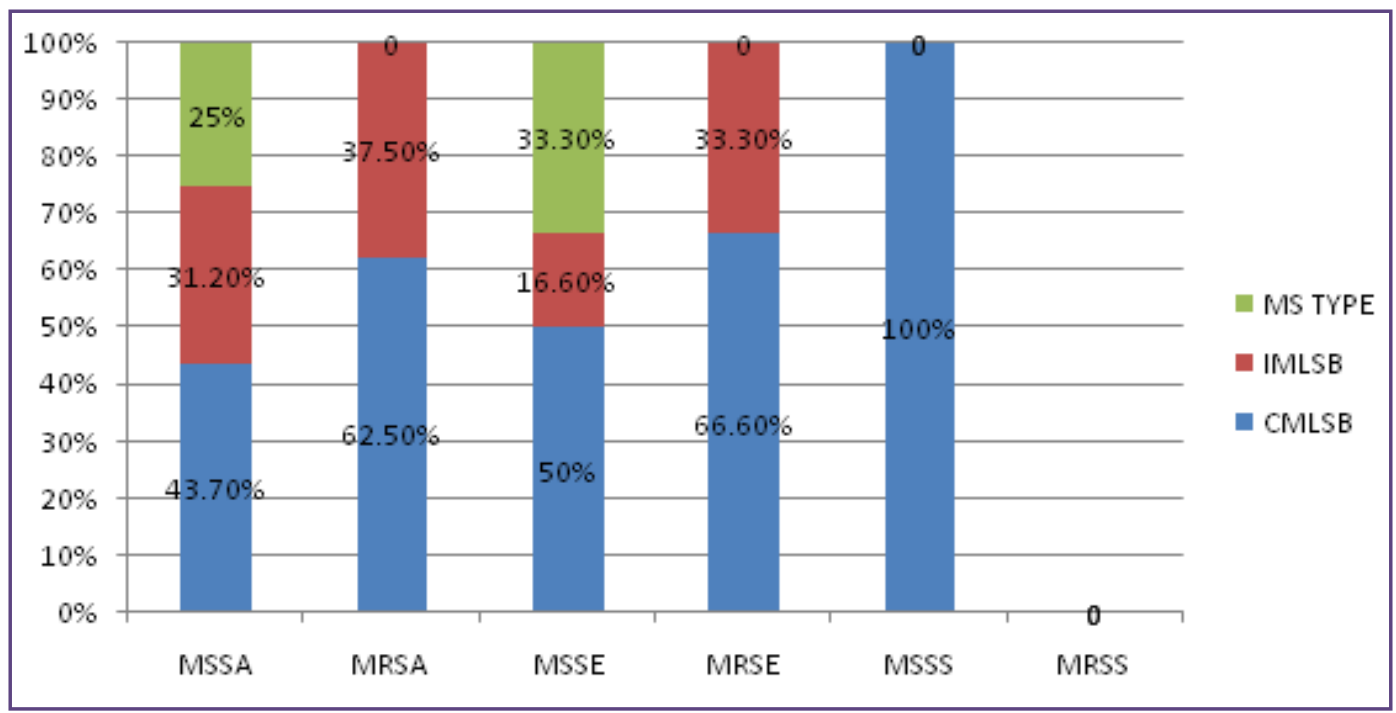

Fig. 2: Distribution of MLSB resistance phenotypes in Staphylococcal isolates.

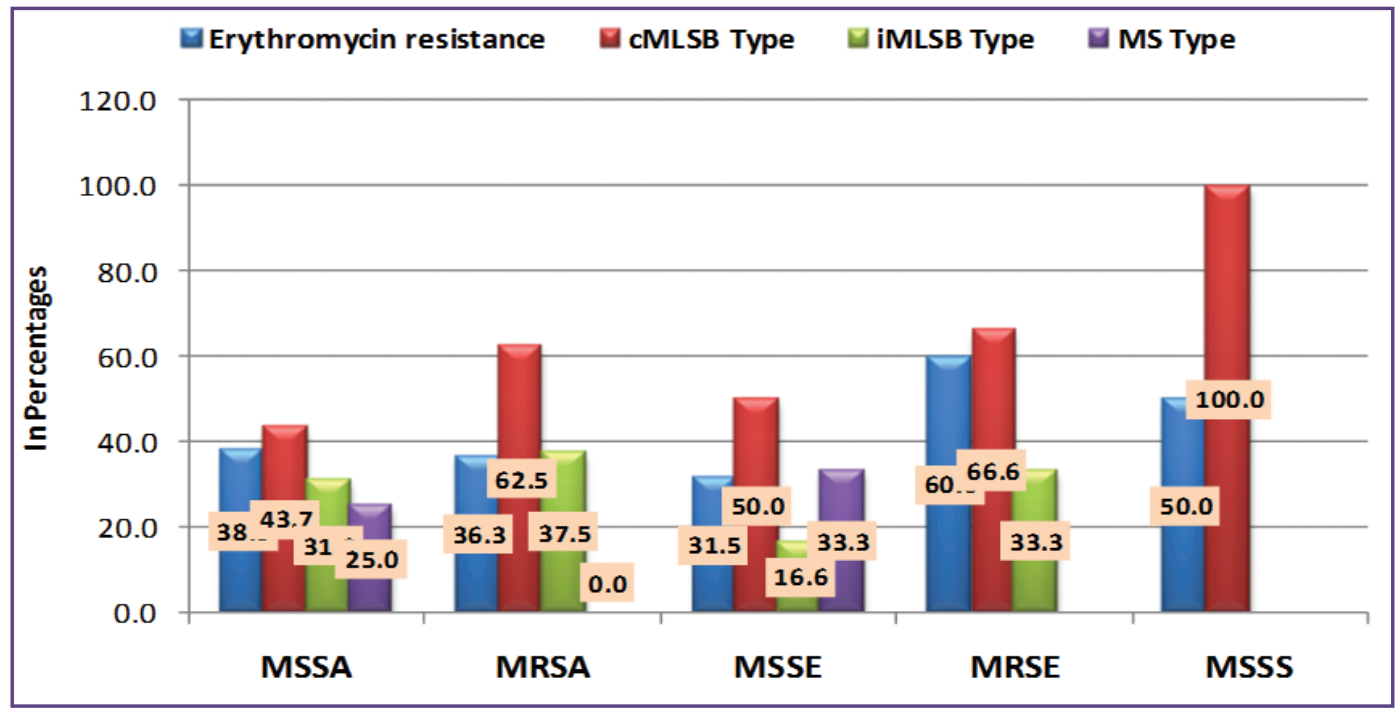

Fig. 3: Association Between type of isolate and type of methicillin resistance.

\section{Discussion}

Out of the 184 Staphylococcal isolates obtained from various sites, $69.5 \%$ were identified as $\mathrm{S}$ aureus and $30.4 \%$ as Coagulase negative Staphylococcus spp. S aureus was the most common isolate in our study.

In addition, methicillin sensitive strains were more common both among $\mathrm{S}$ aureus as well as Coagulase negative staphylococcus spp.In our study $34.3 \%$ of $\mathrm{S}$ aureus isolates were methicillin resistant while $20.8 \%$ of Coagulase negative staphylococcus spp isolates were methicillin resistant. Various Indian studies concluded that MRSA as one of the common cause of hospital-acquired infections and different hospitals have reported a prevalence of about $30 \%-80 \%$ methicillin resistance. ${ }^{[13,14,15]}$ Newer antibiotics like vancomycin, linezolid, and pristinomycin have been advocated in the management of such infections, but recent resistance trends are alarming. ${ }^{[16,17]}$ It raises real concerns over how long these uniform susceptibilities will hold good. In such a scenario, clindamycin becomes a good cost effective choice. Good oral absorption makes this drug an important option in outpatient therapy and as a follow-up after intravenous therapy. ${ }^{[18]}$ In recent times, clindamycin has become the drug of choice for Staphylococcal skin and soft tissue infections as it directly inhibits the Staphylococcal toxin production. Clindamycin attains high concentrations in abscesses and requires no renal dose adjustments .It can be used as an alternative in penicillin-allergic patients. [18] It is effective against both methicillin resistant and the methicillin sensitive Staphylococcal isolates. ${ }^{[5]}$ 
In our study $38.02 \%$ of isolates were resistant to erythromycin. Our findings were supported by studies of Prabhu et al and Deotale $\mathrm{V}$ et al where the prevalence of erythromycin resistance was $28.4 \%$ and $32.4 \%$ respectively. ${ }^{[8,19]}$ The prevalence of constitutive clindamycin resistance was found to be $54.2 \%$ in our study which was higher than what is reported in other studies. ${ }^{[19,20,21]}$ Inducible clindamycin resistance was observed in $28.5 . \%$ of the isolates in our study which was in concordance with the resistance rates of $28.2 \%$ reported by Prabhu et al. ${ }^{[8]}$ However lower (10.5\%) and higher rates $(50.6 \%, 49 \%)$ of inducible clindamycin resistance have been reported by other authors. ${ }^{[8,10,22]}$ Our observations suggest that skipping the D test will lead to nearly one third of the erythromycin resistant isolates misidentified as clindamycin sensitive resulting in therapeutic failure. Misreporting of Staphylococcal isolates susceptible to clindamycin without checking for inducible resistance may result in institution of inappropriate clindamycin therapy. Not performing D test would underestimate clindamycin resistance. On the other hand, negative result for inducible clindamycin resistance confirms clindamycin susceptibility.

The constitutive clindamycin resistance was found to be higher in MRSA and Methicillin resistant S epidermidis (MRSE) as compared to MSSA and Methicillin sensitive $\mathrm{S}$ epidermidis (MSSE) (62.5\% and 66.6\% vs $43.7 \%$ and $50 \%)$. This is in concordance with findings by Azap et al (64\% vs $4.6 \%)$, Yilmaz et al (44.2\% vs $4.5 \%)$, Gupta et al (46\% vs1 $0 \%$ ) , and Farooq $\mathrm{S}$ et al (49.52\% vs $8.27 \%$ ) who reported a higher prevalence of $\mathrm{c} \mathrm{MLS}_{\mathrm{B}}$ phenotype in MRSA as compared to MSSA. ${ }^{[21,23,24,25]}$ Constitutive mutants can be selected in vitro in the presence of clindamycin or any other non-inducer macrolide as they are widespread among methicillin-resistant strains. ${ }^{[26]}$

Similarly, the inducible clindamycin resistance was higher in MRSA and MRSE as compared to MSSA and MSSE (38\% and $33.3 \%$ vs $31.2 \% \%$ and $16.6 \%$ ) .This was in concordance with few other studies. ${ }^{[24,27,20]}$

In studies by Yilmaz et al, Braun et al and Gadepalli et al inducible resistance to clindamycin in MRSA isolates was found to be $24.4 \%, 30 \%$ and $30 \%$ respectively. ${ }^{[24,27,20]}$ However lower prevalence of inducible resistance to clindamycin in MRSA has also been reported in literature. ${ }^{[23,28]}$ High frequency (63\%) of iMLS B resistance is reported among S. aureus isolates with an erythromycin-resistant and clindamycin-susceptible phenotype. Inducible MLS
B phenotypes were more in MRSA (74\%) as compared to MSSA (45\%). ${ }^{[29]}$ This indicates that the prevalence of resistance phenotypes vary among methicillin sensitive and methicillin resistant staphylococcal isolates among different geographical regions. Induced clindamycin resistant Staphylococcal spptend to be multidrug resistant because of the overlapping binding sites of macrolides, lincosamides, and streptogramins $\mathrm{B}$ in $23 \mathrm{~S}$ rRNA. This accounts for cross-resistance amongst the three classes of drugs. ${ }^{[30]}$ Such a situation limits treatment options and increases the likelihood of inadequate antimicrobial therapy.

We observed a high prevalence of MS phenotypes $(25 \%$ and $33.3 \%$ ) in our study among MSSA and MSSE isolates respectively. This could be attributed to the empirical and inappropriate use of macrolides. Macrolides are frequently used over the counter and usage for non bacterial infections leads to selection of resistant mutants. Hence it is emphasized that macrolide antibiotics should always be used with caution during empirical therapy. Also, due to the shared site of activity, MLS group of drugs can be antagonistic to each other and lincosamides should not be administered concurrently with erythromycin.

Appropriate therapy decisions demands vigilance on the resistance trends. The pattern of macrolide resistance in Staphylococcus spp varies in different regions. This makes prescription patterns for clindamycin very variable. There is no substantial data regarding clindamycin prescription from India. ${ }^{[5,8]}$ It is kept as a reserve drug and is usually advocated in severe in-patient MRSA infections depending upon the antimicrobial susceptibility testing. The patients attending our hospital are generally financially burdened. Thus by prescribing clindamycin, use of reserve antibiotics can be spared. However, expression of inducible resistance to clindamycin could limit its effectiveness. Inducible resistance is often missed by the clinical microbiologists in routine testing when erythromycin and clindamycin discs are placed in nonadjacent positions. So, clinical microbiology laboratories should correctly report inducible clindamycin resistance in Staphylococcus spp, and D-test can be used as a simple, cost effective and reliable method to delineate inducible and constitutive clindamycin resistance in routine clinical laboratories.

\section{Conclusions}

We recommend that the clinical microbiology laboratories should test inducible clindamycin resistance by $\mathrm{D}$ test for all isolates that appear erythromycin resistant and clindamycin susceptible in vitro to avoid therapeutic 
failure. This will also prevent an underestimated clindamycin resistance rate in different geographic regions. Macrolides should be used with caution as an empirical therapy. Clindamycin should not be administered concurrently with erythromycin as these drugs can be antagonistic. The prevalence of both constitutive as well as inducible clindamycin resistance is higher in methicillin resistant strains which limit the treatment options and demands prompt vigilance of resistance trends. Prevalence of macrolide resistant phenotypes should be evaluated in CA MRSA and HA-MRSA strains in our community especially in the background of high constitutive resistance when selection of resistant mutants can be fast. Our study gives a spectrum of clindamycin resistance among clinical isolates of $S$. aureus from this region of the country which will help clinicians choose an appropriate therapy.

\section{References}

1. Timothy Foster. Staphylococcus. In: Medical Microbiology; $4^{\text {th }}$ edition: Galveston (TX): University of Texas Medical Branch at Galveston;1996.p.1-12.

2. Watkins RR, David MZ, Salata RA. Current concepts on the virulence mechanisms of methicillin resistant Staphylococcus aureus. J Med Microbiol. 2012;61:1179-93.

3. Sasirekha B, Usha MS, Amruta JA, Ankit S, Brinda N, DivyaR.Incidence of constitutive and inducible clindamycin resistance among hospital-associated Staphylococcus aureus. 3 Biotech. 2014;4(1):85-89.

4. Vivek JS, Rajesh GN, Mukesh S, Manpreet K, Misra RN, Matnani GB et al. The prevalence of inducible clindamycin resistance among community-and hospital-associated Staphylococcus aureus isolates in a tertiary care hospital in India. Biomedical Res 2011; 22:465-469.

5. Juyal D, Shamanth AS, Pal S, Sharma MK, Prakash $\mathrm{R}$, Sharma N.The prevalence of inducible clindamycin resistance among staphylococci in a tertiary care hospital - a study from the garhwal hills of uttarakhand, India. JClinDiagn Res. 2013;7(1):61-5.

6. Mokta KK et al., Inducible Clindamycin Resistance among Methicillin Resistant and Methicillin Susceptible Strains of Staphylococcus aureus. Journal of Clinical and Diagnostic Research. 2015,9(8): p20-23.

7. Leclercq R. Mechanisms of Resistance to Macrolides and Lincosamides: Nature of the Resistance Elements and Their Clinical Implications .Clin Infect Dis.2002: 482-492.

8. Prabhu K, Rao S, Rao V. Inducible Clindamycin Resistance in Staphylococcus aureus Isolated from Clinical Samples. J Lab Physicians. 2011; 3(1): 25-27.

9. Vandana KE, Singh J, Chiranjay M, Bairy I. Inducible Clindamycin Resistance in Staphylococcus aureus: Reason for Treatment Failure. J Glob Infect Dis. 2009;1(1): 76-77.
10. Goyal R, Singh N P, Manchanda V, Mathur M. Detection of clindamycin susceptibility in macrolide resistant phenotypes of Staphylococcus aureus. Indian J Med Microbiol 2004;22:251-4.

11. Clinical and Laboratory Standards Institute. Performance standards for antimicrobial Susceptibility testing, 17th informational supplement (M100-517). 2015, Wayne Pa: Clinical and Laboratory Standards Institute.

12. Baird D. Staphylococcus:Cluster forming Gram positive cocci. In: Mackie \& McCartney, Practical Medical Microbiology. 14th edition. pp245-261.

13. Blomquist PH. Methicilli resistant staphylococcus aureus infections of the eye and orbit (an american ophthalmological society thesis). Trans Am Ophthalmol Soc. Dec; 104: 322-345.

14. Pramodhini S., Thenmozhivalli PR, Selvi R., Dillirani V., Vasumathi A., Agatha D. comparison of various phenotypic methods and mec A based PCR for the detection of MRSA. Journal of Clinical and Diagnostic Research , 2011; 5:1359-1362.

15. Brumfitt W, Hamilton-Miller J. Methicillin-resistant Staphylococcus aureus. N. Engl. J. Med. 1989; 320:1188-1196.

16. Tarai B, Das P, Kumar D. Recurrent Challenges for Clinicians: Emergence of Methicillin-Resistant Staphylococcus aureus, Vancomycin Resistance, and Current Treatment Options. J Lab Physicians. 2013 Jul-Dec; 5(2): 71-78.

17. Kaur DC, Chate SS. Study of Antibiotic Resistance Pattern in Methicillin Resistant Staphylococcus Aureus with Special Reference to Newer Antibiotics. J Glob Infect Dis. 2015 Apr-Jun; 7(2): 78-84.

18. Rayner C, Munckhof WJ. Antibiotics currently used in the treatment of infections caused by Staphylococcus aureus. . Intern Med J. 2005;35Suppl 2:S3-16.

19. Deotale V, Mendiratta DK, Raut U, Narang P. Inducible clindamycin resistance in Staphylococcus aureus isolated from clinical samples. Ind J Med Microbiol. 2010;28:124-6.

20. Gadepalli R, Dhawan B, Mohantay S, Kapil A, Das BK, Chaudhary R. Inducible clindamycin resistance in clinical isolates of Staphylococcus aureus. Indian J Med Res. 2006;123:571-73.

21. Gupta V, Datta P, Rani H, Chander J. Inducible clindamycin resistance in Staphylococcus aureus: A study from north india. J Postgrad Med. 2009;55:176-79.

22. Ajantha GS, Kulkarni RD, Shetty J, Shubhada C, Jain P. Phenotypic detection of inducible clindamycin resistance among Staphylococcus aureus isolates by using the lower limit of recommended inter-disk distance. Indian J PatholMicrobiol 2008;51:376-8.

23. Azap OK, Arslan H, Timurkaynak F, Yapar G, Oruc E, Gagir U. Incidence of inducible clindamycin resistance in erythromycin-resistant isolates of Staphylococcus aureus. J ClinMicrobiol. 2005;43:1716-24. 
24. Yilmaz G, Aydin K, Iskender S, Calyan R, Koksal I. Detection and prevalence of inducible clindamycin resitance in staphylococci. J med Microbiol. 2007;56:342-45.

25. Farooq S, Saleem M. Prevalence of constitutive and inducible clindamycin resistance among clinical isolates of staph aureus in Kashmir valley: a hospital based study. J. Evolution Med. Dent. Sci. 2016; 5(17):828-831.

26. Ciraj A M, Vinod P, Sreejith G, Rajani K. Inducible clindamycin resistance among clinical isolates of staphylococci. Indian J PatholMicrobiol 2009;52:49-51.

27. Braun L, Craft D, Williams R, Tuamokumo F, Ottolini $\mathrm{M}$. Increasing clindamycin resistance among methicillin resistant Staphylococcus aureus in 57 northeast United
States military treatment facilities. Pediatr Infect Dis J 2005;24:622-6.

28. Fokas, S. Fokas, M. Tsironi, M. Kalkani, M. Dionysopouloy. Prevalence of inducible clindamycin resistance in macrolideresistant Staphylococcus spp.ClinMicrobiol Infect, 11 (2005), pp. 337-340

29. Ajantha GS, Kulkarni RD, Shetty J, Shubhada C, Jain P. Phenotypic detection of inducible clindamycin resistance amongst Staphylococcus aureus isolates by using lower limit of recommended inter-disk distance. Indian J PatholMicrobiol. 2008;51:376-8.

30. Parasa LS, Tumati SR. Prevalence of induced clindamycin resistance in methicillin resistant Staphylococcus aureus from hospital population of coastal Andharapradesh, Southindia. Archives of clinical microbiology. 2011;2( 1).

*Corresponding author:

Dr. Monika Rajani, 19/789 Indira Nagar, Lucknow (India) 226016.

Phone: +91 07755091159

Email: drmrajani@rediffmail.com

Date of Submission : 21.04.2017

Date of Acceptance : 29.08.2017

Financial or other Competing Interests: None.

Date of Publication : 19.12.2017 